2. Andersson C, Innala E, Bäckström T. Acute intermittent porphyria in women: clinical expression, use and experience of exogenous sex hormones. A population-based study in northern Sweden. J Intern Med. 2003;254:176-83.

3. Foran SE, Abel G, Guide to the porphyrias. A historical and clinical perspective. Am J Clin Pathol. 2003;119:S86-93.

4. Barclay N.Acute intermittent porphyria in childhood. A neglected diagnosis? Arch Dis Child. 1974;49:404-6.

5. Rosland JH. Recurrent abdominal pain caused by acute intermittent porphyria. Tidsskr Nor Lageforen. 2001;121:2818-20.

\section{Altered sensorium in a chronic alcoholic: pancreatic encephalo- pathy, Wernicke's encephalopathy or Marchiafava Biganami disease?}

\section{Introduction}

Marchiafava Bignami disease (MBD) is a rare disorder, first described in 1903 by Marchiafava and Bignami and characterized by primary progressive demyelination and necrosis of corpus callosum presenting clinically with multifocal central nervous system signs along with altered mental status and seizures. ${ }^{1}$ The exact pathology leading to MBD has not yet been elucidated. Classically, chronic alcoholism and vitamin deficiency have been implicated. Recently, MBD is hypothesized to result from an autoimmune insult or part of a para-neoplastic syndrome. ${ }^{2,3}$

Here, we present the case of a 33-year old male who had history of chronic alcoholism with acute pancreatitis presenting with MBD.

\section{Case report}

A 33-year old male patient with history of daily intake of upto $150 \mathrm{~mL}$ of alcohol for 20 years presented to the emergency with acute onset confusion, decreased speech, hyporesponsiveness, difficulty in recognizing family members and seizures for 2 days.

6 weeks ago, he complained of severe pain in abdomen, sweating, severe vomiting and agitation after an episode of heavy drinking. After 2 days, he developed ascites, constipation and urinary retention. His serum amylase and serum lipase levels were raised and contrast enhanced computerized tomography (CECT) scan of the abdomen showed the presence of pancreatic necrosis ( $>50 \%$ of pancreatic parenchyma) with presence of necrotic fluid collections in lesser sac and right para-colic gutter.

10 days later, he suddenly developed two episodes of generalized seizures within 15 minutes. After the seizures, he

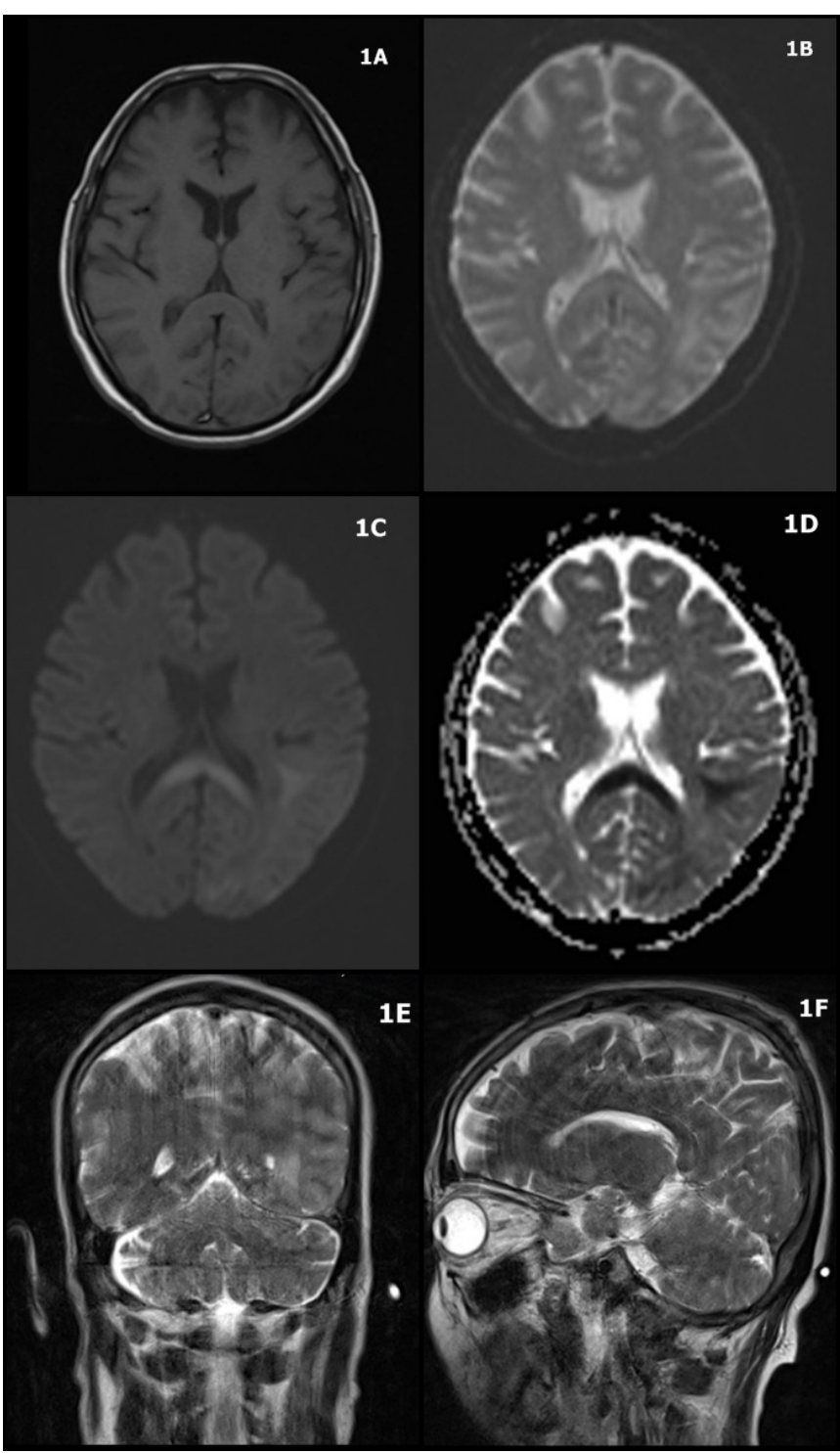

Figure 1: MRI showing lesions in splenium of corpus callosum and peri-ventricular white matter

1A: T1 image showing isointense thickening of splenium; 1B: T2 image showing hyperintense splenium and periventricular white matter on left side

1C: FLAIR showing hyper-intensity in the splenium and white matter on left side

1D: Diffusion weighed image showing restriction in splenu=ium and white matter on left side

1E: T2 showing periventricular hyperintensity on left side

1F: T2 image showing swelling of spleniium along with hyper-intensity 
became unresponsive, drowsy, confused, and disoriented. He was talking irrelevantly, but could gesture urge to defecate and micturate and had to be assisted in doing so.

On the next evening, he was brought to the emergency department of our hospital, where on examination, he was found to be delirious. There was spontaneous eye movement, purposeful movement to painful stimulus and inappropriate responses with discernible words (GCS $4+3+5=12$ ). He had ascites and tender upper abdomen, but was afebrile, with stable vitals without signs of meningeal involvement and focal neurological deficit. Routine blood investigations, electrolytes, HIV, Hepatitis B and C were normal including INR. Ascitic fluid was exudative with elevated amylase (2586U/L). CSF examination (biochemical, microscopy, ADA level, and India Ink preparation) was normal.

Non Contrast CT Scan (NCCT) brain showed mild atrophy. T2 sequence of axial and sagittal images of MRI scan of brain revealed swelling and hyperintensity of splenium of corpus callosum. Hyperintensity in subcortical white matter regions of temporal, occipital and parietal lobes of left side were seen along with periventricular white matter hyper-intensity along left ventricular trigone. This was iso-intense on T1 with effacement of intervening sulci which showed restriction on diffusion weighed imaging (DWI) (Figure 1).

The patient was diagnosed as a case of acute pancreatitis with alcohol dependence syndrome with MBD. He was managed conservatively with i.v. fluids, antimicrobials, analgesics and multivitamins containing thiamine $200 \mathrm{mg}$ / day.

After 2 weeks, clinical improvement was noted with GCS of $15 / 15$, relevant and coherent speech and orientation was partially improved. After 3 weeks, his mental functioning returned to baseline.

\section{Discussion}

This case presented with altered sensorium and seizures with acute pancreatitis and chronic alcoholism. Hence, the differential diagnosis included delirium tremens, fluid electrolyte imbalance, meningo-encephalitis, thromboembolic stroke, pancreatic encephalopathy (PE), Wernicke's encephalopathy (WE) and MBD.

Last alcohol intake being a month ago ruled out delirium tremens, which usually occurs within 48-96 hours after last or reduced intake. Normal serum electrolytes, absence of signs of meningeal irritation and normal CSF examination ruled out delirium due to electrolyte imbalance or meningo-encephalitis.
Thrombo-embolic stroke was ruled out by lack of any focal neurological deficit, CT scan findings and the location of lesions on MRI.

$\mathrm{PE}$ is a common complication of severe pancreatitis, presenting as confusion, restlessness, and associated with diffuse demyelination and white matter changes on cerebral imaging. ${ }^{4}$ Since the white matter changes were not similar to PE, it was excluded.

WE and MBD occur frequently among alcoholics and may co-exist. It is sometimes difficult to differentiate between them clinically. The diagnosis of WE is primarily clinical and presence of any of the two of the following: eye signs, cerebellar signs, dietary deficiency and memory impairment or altered mental functions, has been observed as $85 \%$ sensitive. This patient had altered mental status and possibly dietary deficiency but, cerebellar signs could not be established. However, nystagmus was not seen on examination. Thus, WE could not be ruled out clinically. On the other hand, clinical diagnosis of MBD was supported by altered sensorium, seizures and confusion. ${ }^{1}$

MRI brain can reliably differentiate the two conditions. Lesions in WE associated with alcoholism are bilaterally symmetrical and often involve mamillary bodies, thalamus, tectal plate and peri-aqueductal grey. ${ }^{5}$ Swelling and T2 hyperintensity of splenium of corpus callosum and extracallosal regions with restriction on DWI in this case supported the diagnosis of MBD. ${ }^{5} \mathrm{MBD}$ lesion may extend into genu and adjacent white matter of corpus callosum. MBD is often misdiagnosed as WE, and this might be one reason why involvement of the corpus callosum has been reported in atypical cases of WE. It may also be possible that WE and MBD are etiologically same and lie in a spectrum with involvement of different brain areas giving rise to different clinical features.

Our patient might have developed MBD due to alcohol related neurotoxicity or due to a thiamine deficient state caused by alcohol or severe vomiting. An interesting alternative hypothesis in this case is that the resultant pro-inflammatory state created by the acute pancreatitis might have resulted in the MBD by means of a yet unknown cascade of immunogenic mechanism

The diagnosis and prevalence of MBD may increase with increasing use of routine neuroimaging all over the world. MBD should be kept as a differential diagnosis in all cases of WE and in those with chronic alcoholism and/or inflammatory conditions presenting with altered sensorium and cognitive impairment. 
ROHIT GUPTA ${ }^{1}$, SOURAV DAS ${ }^{2}$, RAVI GUPTA2, VIVEK AHUJA ${ }^{1}$,

MANJU SAINI ${ }^{3}$, MOHAN DHYANI ${ }^{2}$

Correspondence: Dr Rohit Gupta Departments of Gastroenterology ${ }^{l}$ Psychiatry \& Sleep Clinic ${ }^{2}$, Radiodiagnosis ${ }^{3}$ Himalayan Institute of Medical Sciences, Swami Ram Nagar, Doiwala Dehradun 248140, India Email:doctorrohitgupta@yahoo.co.in

\section{References}

1. Hoshino Y, Ueno Y, Shimura H, Miyamoto N, Watanabe M, HattoriN et al. Marchiafava-Bignami disease mimics motor neuron disease: case report. BMC Neurology. 2013;13:208.

2. Celik Y, Temizoz O, Genchellac H, Cakir B, Asil T. A non-alcoholic patient with acute Marchiafava-Bignami disease associated with gynecologic malignancy: paraneoplastic Marchiafava-Bignami disease? Clin Neurol Neurosurg. 2007;109:505-8.

3. Furukawa K, Maeshima E, Maeshima S, Ichinose M. Multiple symptoms of higher brain dysfunction caused by MarchiafavaBignami disease in a patient with dermatomyositis. Rheumatol Int. 2011;31:109-12.

4. Zhang XP, Tian H. Pathogenesis of pancreatic encephalopathy in severe acute pancreatitis. Hepatobiliary Pancreat Dis Int. 2007;6:134-40.

5. Bano S, Mehra S, Yadav SN, Chaudhary V. Marchiafava-Bignami disease: Role of neuroimaging in the diagnosis and management of acute disease. Neurol India. 2009;57:649-52.

\section{Chylous ascitis post primary living donor liver transplantation managed by dietary modification}

\section{Introduction}

Chylous ascites is an intraperitoneal collection of milky fluid caused by blocked or disrupted lymph flow through chyletransporting vessels. It is a rare complication of surgical intervention, and the most common causes are abdominal malignancy and abdominal aortic surgery. Chylous ascites after liver transplantation (LT) is an extremely rare complication, the reported incidence of chylous ascites after LT varies from $0.6 \%$ to $4.7 \%$. Here we report the successful use of dietary modification to treat a case of chylous ascites after living donor liver transplantation (LDLT),

\section{Case report}

A four-month old female baby was brought to the hospital with complaints of jaundice for three weeks along with passage of clay colored stools and dark urine. The birth weight of the child was $2.8 \mathrm{~kg}$. There was no history of fever, bleeding or any abnormal movements, or poor weight gain. The child was investigated at her local hospital and was noticed with cholestatic jaundice with bilirubin of 7.4 (total), 5.2 (direct). USG abdomen done revealed triangular cord sign, HIDA done was non-excretory with good uptake. At the time of presentation to this health care facility at age of 4 months her weight was 4.5 $\mathrm{kg}\left(<5^{\text {th }}\right.$ percentile $)$ and height $59 \mathrm{~cm}\left(<5^{\text {th }}\right.$ percentile $)$. Examination revealed icterus with $6 \mathrm{~cm}$ hepatomegaly and $3 \mathrm{~cm}$ splenomegaly with no free fluid in the abdomen. Her total serum bilirubin was $8.9 \mathrm{mg} / \mathrm{dL}$ with direct fraction of $5.2 \mathrm{mg} / \mathrm{dL}$, ALT was $210 \mathrm{IU} / \mathrm{mL}$, AST $200 \mathrm{IU} / \mathrm{mL}$, GGT 290. Liver biopsy showed bile duct proliferation, bile plugs along with findings of biliary cirrhosis. In view of cirrhotic changes seen on biopsy, Kasai portoenterostomy was deferred and the parents were counseled for a primary liver transplant. She was monitored monthly for growth and development. She was started on calorie dense nasogastric feeds for optimizing her growth along with other medical measures for cholestasis. At age of 7 months she underwent a primary living donor liver transplantation (LDLT), at weight of $6.0 \mathrm{~kg}\left(<5^{\text {th }}\right.$ percentile $)$ and height of $62 \mathrm{~cm}\left(<5^{\text {th }}\right.$ percentile) due to growth failure and deteriorating liver functions. Her mother was the donor. Reduction of the graft (left lateral segment) was done along the superolateral and inferolateral surfaces from 204 grams to 182 grams. The surgery was uneventful and the patient was transferred to the intensive care unit, where she remained hemodynamically stable, and was extubated on the second postoperative day and started on enteral feeds on $6^{\text {th }}$ day postoperative. Immunosuppressive regimen was initiated in the intra and post-operative period. The immediate graft function was excellent with rapidly improving liver function tests. After 48 hours of feeds her drain became cloudy and milky (Figure 1). Drain triglyceride sent were $438 \mathrm{mg} / \mathrm{dL}$. Diagnosis of chylous ascitis was made and she was started on fat free diet with supplementation of medium chain triglyceride, adequate proteins were added $(1.0 \mathrm{~g} / \mathrm{kg})$ along with multivitamins. After 8 days her drain color started improving with reduction in the drain amount, drain triglyceride done on day 8 was $350 \mathrm{mg} / \mathrm{dL}$. Drain triglycerides were monitored; there was significant reduction in drain output and triglyceride contents with the dietary modification (Figure 2). The drain was removed on post-operative day 30 ; at the time 